\title{
Estudo da circulação retrobulbar e do campo visual após dose única oral de citrato de sildenafil (Viagra ${ }^{\circledR}$ )
}

\author{
The effects of a single dose of sildena fil citrate $\left(\right.$ Viagra $\left.^{\circledR}\right)$ on the retrobulbar circulation \\ and visual field
}

\author{
Alessandra Kurahashi ${ }^{1}$ \\ Maurício Nascimento ${ }^{1}$ \\ Ana Maria Marcondes ${ }^{2}$ \\ Nelson Macchiaverni Filho ${ }^{2}$ \\ Jamal Baracat ${ }^{3}$ \\ Vital Paulino Costa ${ }^{4}$
}

\begin{tabular}{|c|}
\hline RESUMO \\
\hline $\begin{array}{l}\text { Objetivo: Estudar os efeitos na circulação retrobulbar e no campo visual } \\
\text { de uma dose oral única de } 100 \text { mg de citrato de sildenafil (Viagra }{ }^{\circledR} \text { ). } \\
\text { Métodos: Um estudo duplo-mascarado e controlado por placebo foi } \\
\text { realizado em } 10 \text { voluntários do sexo masculino, com idade média de } 27,7 \\
\pm 5,68 \text { anos. O olho direito de cada voluntário foi submetido aos exames } \\
\text { de Doppler colorido de órbita e análise de campo visual por meio de } \\
\text { perimetria computadorizada (Humphrey, programa 30-2, estratégia "Full } \\
\text { Threshold") em } 3 \text { ocasiões: "baseline", } 1 \text { hora após placebo e } 1 \text { hora após } \\
100 \text { mg de sildenafil via oral. No campo visual, analisaram-se o limiar } \\
\text { foveal e o "mean deviation" (MD) nas } 3 \text { ocasiões. No Doppler colorido, } \\
\text { medimos a velocidade sistólica máxima (VSM), a velocidade diastólica } \\
\text { final(VDF) e o índice de resistência (IR) da artéria central da retina (ACR) } \\
\text { e da artéria oftálmica (AO) nas } 3 \text { ocasiões. Resultados: A administração } \\
\text { do sildenafil não alterou significativamente o limiar foveal e o "mean } \\
\text { deviation" em relação ao "baseline" e ao placebo. Houve um aumento } \\
\text { significativo da velocidade sistólica máxima e velocidade diastólica final } \\
\text { na artéria oftálmica após a administração do citrato de sildenafil (p<0,001). } \\
\text { Não houve alteração significativa dos parâmetros hemodinâmicos na } \\
\text { artéria central da retina e no índice de resistência da artéria oftálmica. } \\
\text { Conclusões: A administração de } 100 \text { mg de citrato de sildenafil promoveu } \\
\text { um aumento da velocidade de fluxo sanguíneo na artéria oftálmica de } \\
\text { indivíduos normais e não promoveu alteração significativa no limiar } \\
\text { foveal e "mean deviation" da campimetria computadorizada. }\end{array}$ \\
\hline
\end{tabular}

Descritores: Artéria retiniana/efeitos de drogas; Campos visuais/efeitos de drogas; Piperazinas/administração e dosagem; Velocidade do fluxo sanguíneo; Ultra-sonografia Doppler em cores; Fóvea central; Dose única; Método duplo-cego

\section{INTRODUÇÃ̃O}

O citrato de sildenafil (Viagra ${ }^{\circledR}$, UK-92,480) é um medicamento indicado para o tratamento da disfunção erétil peniana, condição clínica significativa e comum que afeta milhões de homens no mundo todo. É um inibidor potente e seletivo da enzima fosfodiesterase tipo 5 (PDE5), específica para o monofosfato de guanosina cíclico (GMPc), encontrado em concentrações relativamente altas nos tecidos do corpo cavernoso e nas plaquetas. A PDE5 se encontra também na musculatura esquelética, musculatura lisa vascular e visceral ${ }^{(1-2)}$.

Seis isoenzimas PDE foram identificadas e bem caracterizadas, cada uma com diferentes propriedades e distribuições tissulares em seres humanos e em

Residentes de Oftalmologia do Hospital de Clínicas da

Chefe do Setor de Glaucoma do Departamento de Oftalmologia da Faculdade de Ciências Médicas (FCM) Uni- 
animais. A isoforma PDE6 é encontrada nos fotorreceptores e media o processo de transdução através do controle do nível de GMPc na retina. Apesar de ser seletiva para a PDE5, o sildenafil tem um efeito inibitório leve sobre a PDE6, podendo causar visão borrada e azulada, ou aumento da sensibilidade à luz ${ }^{(3-4)}$. Não foram registradas lesões retinianas tardias, porém não foram realizados estudos eletrorretinográficos de maior duração ${ }^{(3)}$.

A avaliação dos efeitos hemodinâmicos do sildenafil foi objeto de vários estudos. A administração de doses únicas orais de sildenafil de até $100 \mathrm{mg}$ a voluntários sadios não produziu efeitos clinicamente significantes no eletrocardiograma ${ }^{(3)}$. A média da diminuição da pressão arterial sistólica na posição supina, após administração oral de $100 \mathrm{mg}$, foi de $8,4 \mathrm{mmHg}^{(1)}$. A mudança correspondente na pressão arterial diastólica na posição supina foi de 5,5 mmHg. Essas diminuições na pressão arterial são consistentes com os efeitos vasodilatadores do sildenafil, provavelmente secundários ao aumento dos níveis de GMPc na musculatura lisa dos vasos sanguíneos. Sob condições fisiológicas, os efeitos do sildenafil sobre a PA são de natureza leve a moderada e normalmente não associadas a sintomas, exceto cefaléia leve e rubor facial. Entretanto, nos pacientes que recebem terapia com nitratos, o sildenafil pode potencializar o efeito vasodilatador do óxido nítrico resultando em queda significativa da $\mathrm{PA}^{(5)}$. Jackson et al. observaram alterações circulatórias moderadas após injeção endovenosa ou endoarterial de sildenafil, porém não constataram mudanças significativas nos índices cardíacos após administração oral ${ }^{(6)}$.

Eventos vasculares retinianos já foram descritos em usuários de citrato de sildenafil, porém sua incidência não ultrapassou a esperada para a população idosa ${ }^{(3)}$. Também há relatos de perda de visão temporária em homens com problemas cardiovasculares que fizeram uso de sildenafil, o que parece estar mais relacionado à doença cardiovascular de base do que a um efeito direto da medicação no olho ${ }^{(7)}$.

Apesar de não alterar a pressão intra-ocular ${ }^{(3)}$, o sildenafil pode alterar o fluxo vascular e volume coroidal através de sua ação na musculatura lisa da parede vascular. Dado o potencial de alterações hemodinâmicas associado ao uso do citrato de sildenafil, decidiu-se analisar a influência da droga na circulação retrobulbar, através do Doppler colorido de órbita e no campo visual, através de perimetria computadorizada em voluntários do sexo masculino após dose única oral.

\section{MÉTODOS}

Este estudo foi desenvolvido pelos Departamentos de Oftalmologia e Radiologia do Hospital de Clínicas da Universidade Estadual de Campinas - UNICAMP. O estudo foi aprovado pelo Comitê de Ética em Pesquisa da FCM-UNICAMP.

Foram estudados os efeitos na circulação retrobulbar e no campo visual após uma dose oral única de 100mg de Sildenafil. A casuística foi composta por 10 voluntários do sexo masculino, com idade média de 27,7 $\pm 5,68$ anos (variando de 23 a 38 anos). O estudo foi duplo-mascarado, controlado com place- bo. O olho direito de cada voluntário $(n=10)$ foi submetido aos exames de Doppler colorido de órbita e perimetria computadorizada em três ocasiões distintas com intervalo de 1 semana: "baseline", 1 hora após placebo e 1 hora após a ingestão de $100 \mathrm{mg}$ de sildenafil. A perimetria computadorizada foi realizada imediatamente após o Doppler.

Para realização do exame de Doppler colorido, o Hospital das Clínicas da UNICAMP dispõe do equipamento Ultramark 9 - modelo HDI fabricado pela ATL. O transdutor utilizado neste estudo foi o linear de freqüência variável (6 a $9 \mathrm{MHz}$ ). A avaliação foi realizada por um único examinador (VPC), mascarado para a susbstância utilizada pelo voluntário.

O paciente era colocado em decúbito dorsal horizontal. Com os olhos fechados, colocava-se o gel de contato sobre a pálpebra superior do olho a ser examinado, seguido do transdutor linear. A imagem ecográfica em tempo real funciona como guia anatômico, orientando a localização dos vasos orbitários. Estes são mais facilmente visualizados graças a um código de cores que fornece informações qualitativas e quantitativas sobre o fluxo sangüíneo. As informações qualitativas incluem o sentido do fluxo sangüíneo. Em geral, codificamos o movimento de sangue em direção ao transdutor em vermelho e o movimento contrário ao transdutor em azul. Um "scan" horizontal através do globo ocular e da órbita ao nível do nervo óptico permite a visualização do complexo artéria/veia central da retina, que pode ser detectado nos $3 \mathrm{~mm}$ anteriores da "sombra" correspondente ao nervo óptico. A análise espectral deste complexo revela um componente arterial (pulsátil e positivo, acima da linha horizontal) e um componente venoso (laminar, negativo e abaixo da linha horizontal). A artéria oftálmica pode ser visualizada profundamente na órbita, onde ocupa uma posição temporal ao nervo óptico, ou mais anteriormente, após cruzamento sobre o nervo. O perfil espectral da artéria oftálmica é típico, apresentando um "notch" dicrótico durante a sístole e velocidades maiores que as observadas nos outros vasos ${ }^{(8)}$.

Quanto à análise quantitativa, a visualização dos vasos permite a determinação do ângulo $\theta$ e, consequentemente, a velocidade do fluxo sanguíneo (como na equação Doppler). Uma vez visualizado o vaso e corrigido o valor do ângulo $\theta$, congela-se a imagem e procede-se à análise espectral do fluxo. Baseado na análise do perfil espectral, pode-se calcular a velocidade sistólica máxima (VSM) e a velocidade diastólica final (VDF) naquele vaso. Indiretamente, podemos calcular índices de resistência, dos quais o mais utilizado é o índice de Pourcelot, calculado como: VSM-VDF/VSM. O índice de Pourcelot é diretamente proporcional à resistência ao fluxo sangüíneo em um determinado vaso ${ }^{(8)}$.

Neste estudo, analisamos a VSM, a VDF e o índice de resistência da artéria central da retina (ACR) e da artéria oftálmica nas 3 ocasiões.

A análise do campo visual foi realizada através de perimetria computadorizada Humphrey (Humphrey Instruments Inc, San Leandro, EUA), utilizando o programa central 30-2 e a estratégia Full Threshold. O teste consta da pesquisa de 76 pontos dispostos nos 30 graus centrais do campo visual. A distância entre os pontos analisados é de 6 graus, a duração 
do estímulo é de 0,2 segundos e o tamanho do estímulo utilizado é equivalente ao estímulo III do perímetro de Goldmann ${ }^{(9)}$. O limiar foveal e o mean deviation (MD) (média aritmética dos valores do gráfico numérico do total deviation) foram os parâmetros perimétricos avaliados nas 3 ocasiões.

Utilizou-se o teste de análise de variância para medidas repetidas. Valores de $\mathrm{p}<0,05$ foram considerados significantes.

\section{RESULTADOS}

A tabela 1 revela que a administração do sildenafil não alterou significativamente o limiar foveal e o MD em relação ao baseline e ao placebo (Tabela 1).

A tabela 2 revela a média dos parâmetros hemodinâmicos medidos nos 10 pacientes nas 3 ocasiões. Houve um aumento significativo da VSM e VDF na artéria oftálmica após a administração do citrato de sildenafil $(\mathrm{p}<0,001)$. Não houve alteração significativa dos parâmetros hemodinâmicos na ACR e no IR da artéria oftálmica.

A tabela 3 especifica as variações de velocidades da ACR e artéria oftálmica dos pacientes que utilizaram sildenafil e placebo em relação ao baseline.

\section{DISCUSSÃO}

As reações adversas mais comuns associadas ao tratamento com sildenafil tem sido relacionadas à vasodilatação (incluindo rubor, cefaléia, e congestão nasal) e a efeitos gastrointestinais. Vários autores tem descrito alterações visuais

\begin{tabular}{|c|c|c|c|c|}
\hline Parâmetro & Baseline & Placebo & Sildenafil & $\mathbf{P}$ \\
\hline Fóvea (dB) & $38,10 \pm 1,79$ & $37,30 \pm 1,63$ & $38,60 \pm 1,43$ & 0,086 \\
\hline $\mathrm{MD}(\mathrm{dB})$ & $-1,40 \pm 1,47$ & $-1,30 \pm 1,49$ & $-0,93 \pm 1,76$ & 0,680 \\
\hline
\end{tabular}

associadas ao uso do citrato de sildenafil. Queixas visuais foram relatadas em $2,7 \%$ dos usuários, caracterizadas por alteração predominantemente nas cores, de natureza leve e transitória, além de sensibilidade aumentada à luz e visão borrada. Nesses estudos, apenas um paciente interrompeu o tratamento devido à alteração visual ${ }^{(3)}$. Relatou-se um caso de melhora na deficiência de visão de cores verde-vermelho verificada com diferentes testes de visão de $\operatorname{cores}^{(10)}$.

Utilizando-se o teste de coloração de Farnsworth-Munsell 100, foram observadas em alguns indivíduos alterações transitórias na distinção de cores (azul / verde) 1 hora após a administração de uma dose de $100 \mathrm{mg}$, efeito que desaparecia 2 horas após a ingestão. Os resultados dos estudos com doses de até $200 \mathrm{mg}$ (duas vezes a dose máxima recomendada) demonstraram que o único efeito agudo do sildenafil na visão foi alteração fugaz na discriminação das cores azul verde em alguns indivíduos. O mecanismo aceito para essa alteração na distinção de cores está relacionado à inibição da PDE6, que está envolvida na cascata de fototransdução da retina ${ }^{(1,4)}$. Não foram detectadas alterações clinicamente significativas numa bateria de outros exames para avaliação da função visual, incluindo a acuidade visual (diagrama de Snellen), sensibilidade de contrastes, pressão intra-ocular, tela de Amsler, campos visuais e recuperação do teste de stress à luz ${ }^{(4)}$.

Marmor observou que as medidas da função elétrica retinianas caíram em 30 a $50 \%$, o que persistia por pelo menos 5 horas depois do uso de altas doses do sildenafil ${ }^{(11)}$. Klotz e Vobig analisaram os efeitos retinianos agudos de uma dose única de $100 \mathrm{~g}$ de sildenafil e não observaram alterações na acuidade visual, pressão intra-ocular, campo visual, visão de cores e potencial visual evocado. As medidas do eletrorretinograma (ERG) mostraram redução significante na amplitude das ondas A e B uma hora após a administração da droga, com recuperação completa deste efeito 6 horas mais tarde ${ }^{(4,12)}$.

Donahue et al. ${ }^{(13)}$ relataram um caso de paralisia unilateral de III par sem envolvimento pupilar após uso de citrato de sildenafil e sugeriram que a droga poderia induzir hipotensão sistêmica suficiente para causar disfunção neurológica nos pacientes predispostos à doença microvascular.

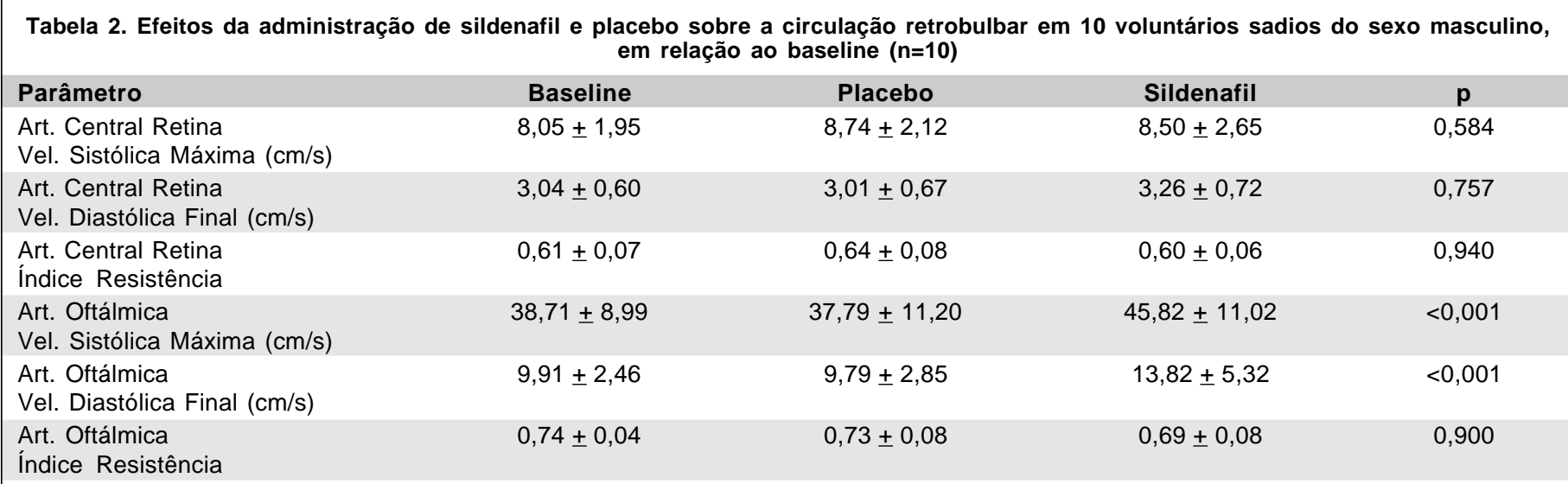




\begin{tabular}{|c|c|c|c|c|c|c|c|c|c|c|c|c|c|c|c|c|c|c|}
\hline & \multicolumn{6}{|c|}{ Baseline } & \multicolumn{6}{|c|}{ Placebo } & \multicolumn{6}{|c|}{ Viagra } \\
\hline & $\begin{array}{c}\text { ACR } \\
\mathrm{S}\end{array}$ & $\begin{array}{c}\text { ACR } \\
\text { D }\end{array}$ & $\begin{array}{c}\text { ACR } \\
\text { RI }\end{array}$ & $\begin{array}{c}\text { AOF } \\
S\end{array}$ & $\begin{array}{c}\text { AOF } \\
\text { D }\end{array}$ & $\begin{array}{c}\text { AOF } \\
\text { RI }\end{array}$ & $\begin{array}{c}\text { ACR } \\
\text { S }\end{array}$ & $\begin{array}{c}\text { ACR } \\
\text { D }\end{array}$ & $\begin{array}{c}\text { ACR } \\
\text { RI }\end{array}$ & $\begin{array}{c}\text { AOF } \\
S\end{array}$ & $\begin{array}{c}\text { AOF } \\
\text { D }\end{array}$ & $\begin{array}{c}\text { AOF } \\
\text { RI }\end{array}$ & $\begin{array}{c}\text { ACR } \\
\text { S }\end{array}$ & $\begin{array}{c}\text { ACR } \\
\text { D }\end{array}$ & $\begin{array}{c}\text { ACR } \\
\text { RI }\end{array}$ & $\begin{array}{c}\text { AOF } \\
S\end{array}$ & $\begin{array}{c}\text { AOF } \\
\text { D }\end{array}$ & $\begin{array}{c}\text { AOF } \\
\text { RI }\end{array}$ \\
\hline Pcte 1 & 8,3 & 3,6 & 0,56 & 39,8 & 9,0 & 0,77 & 6,3 & 2,2 & 0,65 & 33,4 & 8,4 & 0,74 & 6,7 & 3,3 & 0,51 & 54,3 & 22,0 & 0,59 \\
\hline Pcte 2 & 9,4 & 4,2 & 0,55 & 27,4 & 7,6 & 0,72 & 9,2 & 3,1 & 0,66 & 21,8 & 7,7 & 0,64 & 7,2 & 2,8 & 0,61 & 35,7 & 9,1 & 0,74 \\
\hline Pcte 3 & 10,6 & 3,5 & 0,66 & 48,1 & 9,2 & 0,80 & 9,7 & 3,6 & 0,62 & 64,9 & 15,6 & 0,75 & 9,1 & 4,4 & 0,51 & 34,2 & 7,2 & 0,79 \\
\hline Pcte 4 & 5,3 & 2,7 & 0,49 & 53,9 & 15,4 & 0,71 & 9,2 & 2,5 & 0,73 & 55,2 & 10,4 & 0,81 & 8,7 & 3,6 & 0,58 & 43,5 & 13,4 & 0,69 \\
\hline Pcte 5 & 5,7 & 2,1 & 0,63 & 29,6 & 8,4 & 0,71 & 5,7 & 1,9 & 0,66 & 29,0 & 9,0 & 0,68 & 6,3 & 2,5 & 0,60 & 24,9 & 8,0 & 0,67 \\
\hline Pcte 6 & 6,3 & 2,7 & 0,57 & 32,9 & 8,2 & 0,75 & 6,7 & 3,5 & 0,47 & 30,9 & 9,8 & 0,68 & 6,3 & 2,7 & 0,57 & 53,9 & 14,2 & 0,73 \\
\hline Pcte 7 & 7,0 & 2,8 & 0,60 & 36,1 & 12,5 & 0,65 & 9,1 & 4,0 & 0,56 & 33,1 & 13,1 & 0,60 & 5,7 & 2,3 & 0,58 & 41,5 & 19,8 & 0,52 \\
\hline Pcte 8 & 8,5 & 2,7 & 0,68 & 48,4 & 11,3 & 0,76 & 7,9 & 2,7 & 0,66 & 48,0 & 10,7 & 0,77 & 10,5 & 3,6 & 0,66 & 65,2 & 19,7 & 0,69 \\
\hline Pcte 9 & 10,9 & 2,8 & 0,74 & 30,9 & 7,9 & 0,74 & 12,1 & 3,3 & 0,73 & 35,4 & 6,8 & 0,80 & 10,2 & 3,1 & 0,69 & 45,5 & 9,8 & 0,78 \\
\hline Pcte 10 & 8,5 & 3,3 & 0,61 & 40,0 & 9,6 & 0,75 & 11,5 & 3,3 & 0,71 & 36,2 & 6,4 & 0,82 & 14,3 & 4,3 & 0,69 & 49,5 & 15,0 & 0,69 \\
\hline
\end{tabular}

Vários autores relataram a repercussão hemodinâmica ocular do citrato de sildenafil ${ }^{(14-16)}$. Siu et al ${ }^{(14)}$ empregaram a laser Doppler fluxometria e concluíram que não houve mudanças significativas no fluxo sanguíneo na rima do nervo óptico e no fluxo sanguíneo coroidal foveolar após administração de $100 \mathrm{mg}$ do sildenafil. Sugerem que os efeitos dos compostos de nitrato e do sildenafil na circulação ocular devem ser diferentes, uma vez que os compostos de nitrato já provaram ser capazes de aumentar a circulação da cabeça do nervo óptico e de produzir vasodilatação venosa retiniana ${ }^{(15)}$.

Por outro lado, Sponsel et al. ${ }^{(16)}$, ao analisarem o fluxo sanguíneo pulsátil ocular e perimetria computadorizada após dose de $50 \mathrm{mg}$ de sildenafil, observaram um aumento próximo a $30 \%$ no fluxo pulsátil ocular, provavelmente por alterações intrínsecas na vasculatura coroidal com conseqüente impacto positivo na sensibilidade ao contraste.

O Doppler colorido da órbita é um exame não invasivo, não utiliza radiação ionizante e não necessita de alterações artificiais na pressão intra-ocular. Permite avaliação do fluxo sanguíneo retrobulbar, mais especificamente das artérias oftálmica, central da retina e ciliares posteriores curtas. É considerado no diagnóstico e acompanhamento de condições oculares onde exista suspeita da participação de fenômenos vasculares, como nas fístulas carótido-cavernosas, varizes orbitárias, doença oclusiva da artéria carótida, oclusão de artéria ou veia central da retina, neuropatia óptica isquêmica anterior e no glaucoma. Também pode ser utilizado para análise dos efeitos de drogas sobre o fluxo sanguíneo do nervo óptico ${ }^{(8)}$.

O presente estudo confirmou que a dose única de $100 \mathrm{mg}$ de sildenafil não produz alterações significativas no campo visual, avaliado pela perimetria computadorizada. O limiar de sensibilidade foveal e sensibilidade global não se alteraram após a administração da droga. Quanto aos efeitos na circulação retrobulbar, observamos aumento das velocidades de fluxo na artéria oftálmica, provavelmente causado por vasodilatação desses vasos. Apesar do mesmo não ter sido detectado na ACR (o que pode ser consequência do tamanho da nossa amostra), é possível que parte das alterações visuais associadas ao uso do sildenafil possam ser secundários a esta alteração hemodinâmica. No entanto, para comprovar esta hipótese, novos estudos empregando angiofluoresceinografia ou laser Doppler fluxometria se fazem necessários.

\section{ABSTRACT}

Purpose: To analyze the effects of $100 \mathrm{mg}$ of sildenafil citrate $\left(\right.$ Viagra $\left.^{\circledR}\right)$ on the retrobulbar circulation and visual field. Methods: A double masked, placebo controlled study was conducted in 10 males with a mean age of $27.7 \pm 5.68$ years. The right eye of each volunteer underwent orbital color Doppler imaging and automated perimetry (Humphrey, program 30-2, Full-Threshold Strategy) at 3 occasions: baseline, 1 hour after placebo and 1 hour after $100 \mathrm{mg}$ of sildenafil. The foveal threshold and the mean deviation (MD) were analyzed by automated perimetry on the three occasions. Color Doppler imaging allowed the measurement of the peak systolic velocity (PSV), end diastolic velocity (EDV) and Pourcelot index (PI) in the central retinal artery and ophthalmic artery. Results: The foveal threshold and the mean deviation did not show a significant change following the administration of sildenafil. The ophthalmic artery peak systolic velocity and end diastolic velocity significantly increased after the administration of sildenafil $(\mathrm{p}<0.001)$. The hemodynamic parameters in the central retinal artery and the ophthalmic artery PI did not significantly change. Conclusions: Sildefanil citrate increased the blood flow velocities in the ophthalmic artery in normal subjects, with no significant changes in the foveal threshold and mean deviation in the automated perimetry.

Keywords: Retinal artery/drug effects; Visual fields/drug effects; Piperazines/administration \& dosage; Blood flow velocity; Color ultrasonography Doppler; Fovea centralis; Single dose; Double-blind method 


\section{REFERÊNCIAS}

1. Naylor A, Ballard S, Gingell C. Sildenafil (Viagra*): an inhibitor of cyclic GMP - specific phosphodiesterase type 5 for the treatment of male erectile dysfunction. Eur Urol 1996;30:158.

2. Ballard SA, Gingel CJC, Price ME. Sildenafil, an inhibitor of phosphodiesterase type 5, enhances nitric oxide mediated relaxation of human corpus cavernosum [abstract]. Int J Impot Res 1996,8:103.

3. Marmor MF, Kessler R. Sildenafil (Viagra) and ophthalmology. Surv Ophthalmol 1999;44:153-62.

4. Klotz T, Vobig M, Walter P, Heidenreich A, Udo E. Acute retinal side effects of a single dose of $100 \mathrm{mg}$ sildenafil. J Urol 1999;161:215-6.

5. Zusman RM, Morales A, Glasser DB, Osterloh IH. Overall cardiovascular profile of sildenafil citrate. Am J Cardiol 1999;83:35-44.

6. Jackson G, Benjamin N, Jackson N, Allen MJ. Effects of sildenafil citrate on human hemodynamics. Am J Cardiol 1999,83:13-20.

7. Sharpe R. Some Viagra users temporarily lost vision, reports say. Wall St J 1998;B6:12
8. Costa VP. Doppler colorido. In: Abreu G. Ultrassonografia ocular. São Paulo: Cultura Médica; 1996.

9. Costa VP. Perimetria computadorizada: um guia básico de interpretação. Rio de Janeiro: RioMed; 1995. p. 133

10. Wohl E, Tauber R, VonDomarus D, Vorkauf M. Transient improvement of red-green color blindness. Aktuel Urol 1999;30:335-7.

11. American Academy of Ophthalmology. Cautions Viagra users. [announcement] Arch Ophthalmol 1998;116:727-9.

12. Vobig MA. Retinal side-effects of sildenafil [letter]. Lancet 1999;353:1442-3.

13. Donahue SP, Taylor RJ. Pupil-sparing third nerve palsy associated with sildenafil citrate (Viagra). Am J Ophthalmol 1998;126:476-7.

14. Siu K, Grunwald JE, Jacob SS, Du Pont J. Effect of Viagra on ocular circulation [abstract]. Invest Ophthalmol Vis Sci 2000;4:S98.

15. Grunwald JE, DuPont J, Dreyer EB. Effect of chronic nitrate treatment on retinal vessel caliber in open-angle glaucoma [commented on: Am J Ophthalmol 1997;123:817-9]. Am J Ophthalmol 1997;123:753-8.

16. Sponsel WE, Sandoval S, Chen N, Kavanagh J, Khemsera V, Sharma S. Effects of sildenafil (Viagra) on ocular perfusion and contrast sensitivity [abstract]. Invest Ophthalmol Vis Sci 2000;41:S98.

\section{5a ACADEMIA AMERICANA DE OFTALMOLOGIA}

\section{New Orleans - Louisiana - 11 à 14 de novembro de 2001}

O CBO - Conselho Brasileiro de Oftalmologia e a Mello Faro Turismo convidam você a integrar o grupo de profissionais brasileiros que estará presente neste importante evento.

\section{HOSPEDAGEM EM NEW ORLEANS}

\begin{tabular}{l|c|c|}
\multicolumn{1}{|c|}{ HOTEL } & Apartamento Duplo & Apartamento Individual \\
\hline $\begin{array}{l}\text { Holiday Inn Superdome turística superior } \\
330 \text { Loyola Avenue - próximo ao Centro de Convenções }\end{array}$ & USD 250,00 & USD 250,00 \\
$\begin{array}{l}\text { Royal Sonesta } \\
300 \text { Bourbon Street - próximo ao Centro de Convenções }\end{array}$ & USD 286,00 & USD 286,00 \\
\end{tabular}

as diárias iniciam às 15:00h e terminam às 12:00h.

Os valores indicados são por apartamento e por dia com taxas inclusas e estão sujeitos a variações tarifárias até a data da efetiva confirmação da reserva e a variações cambiais até a data do efetivo e total pagamento. As reservas serão aceitas mediante o pagamento de sinal correspondente a USD 200,00 por pessoa a ser abatido do total das diárias utilizadas. O saldo deverá estar integralmente quitado até 20/09/01. Em função dos compromissos assumidos, cancelamentos serão aceitos sem ônus quando solicitados até 01/09/01 - após esta data sujeitam-se à perda do sinal e de outras importâncias já comprometidas com os hotéis, podendo esta perda chegar a $100 \%$ do valor total das diárias.

TRANSPORTE AÉREO as informações sobre vôos serão prestadas durante o atendimento Para o transporte aéreo serão aplicadas tarifas promocionais de excursão com descontos especiais e pagamento parcelado sem juros. Entre as várias opções disponíveis destacamos:

\section{UNITED AIRLINES via Chicago a partir de USD 880,00 por pessoa}

Valor para embarque em São Paulo no dia 09/11/01 e retorno para São Paulo no dia 15/11/01, com possibilidade de embarque e retorno a partir de outras cidades e alteração de datas segundo o interesse de cada participante. Sujeito a disponibilidade de lugares e revisão tarifária. O pagamento pode ser parcelado sem juros através de cartões de crédito segundo as condições vigentes na época. Taxas aeroportuárias não incluídas. As tarifas especiais indicadas serão aplicadas desde que haja um grupo mínimo de 15 pessoas viajando juntos na mesma cia aérea.

\section{PROGRAMAS OPCIONAIS}

A Mello Faro Turismo está à disposição para outros serviços eventualmente necessários tais como traslados, passeios, locação de automóvel, hotéis alternativos e roteiros opcionais para extensão ou complementação da viagem segundo o interesse de cada participante.

Maiores informações e reservas: Mello Faro Turismo e Câmbio Ltda

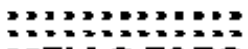

tel 011258.5044 - fax 011231.1343 Email: eventos@mellofaro.com.br

Valores referentes a 07/2001 